\title{
Efficacy of goniosynechialysis for advanced chronic angle-closure glaucoma
}

This article was published in the following Dove Press journal:

Clinical Ophthalmology

26 October 2012

Number of times this article has been viewed

\section{Guoping Qing ${ }^{1,2}$ \\ Ningli Wang' \\ Dapeng $\mathrm{Mu}^{\mathrm{I}}$}

'Beijing Tongren Eye Center, Beijing Tongren Hospital, Capital Medical University, Beijing Ophthalmology and Visual Sciences Key Lab, Beijing, China; ${ }^{2}$ State Key Laboratory of Brain and Cognitive Science, Institute of Biophysics, Chinese Academy of Sciences, Beijing, China

Video abstract

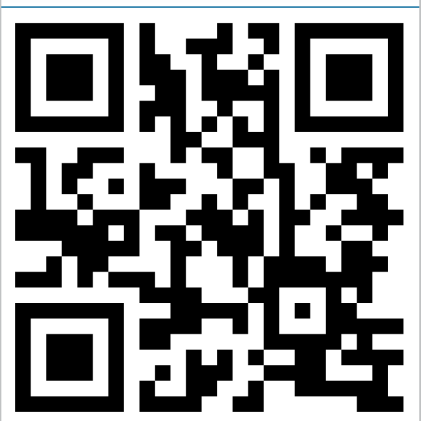

Point your SmartPhone at the code above. If you have a QR code reader the video abstract will appear. Or use: http://dvpr.es/QmteUG

Correspondence: Guoping Qing

No I, Dongjiaominxiang, Dongcheng

District, Beijing, 100730, China

Tel +86 I0 6717 2733

Fax +86 I0 58269920

Email gptsing@।26.com
Purpose: To evaluate the intraocular pressure (IOP)-lowering efficacy of goniosynechialysis (GSL) for advanced chronic angle-closure glaucoma (CACG) using a simplified slit-lamp technique.

Patients and methods: Patients with CACG with one severely affected eye with best-corrected visual acuity below 20/200 and a mildly or functionally unaffected fellow eye were enrolled in this study. All patients underwent ophthalmologic examinations including measurement of visual acuity, best-corrected visual acuity, and IOP; biomicroscopy; specular microscopy; fundus examination; and gonioscopy followed by anterior chamber paracentesis and GSL for nasal peripheral anterior synechiae in the eye with severe CACG.

Results: Thirty patients (18 men, 12 women) were identified as having CACG with an initial mean IOP of $47.1 \pm 6.7 \mathrm{mmHg}$ (range 39-61 $\mathrm{mmHg}$ ) in the severely affected eye. One week after GSL, the mean IOP of the treated eyes decreased to $19.3 \pm 2.8 \mathrm{mmHg}$ (range 14-26 $\mathrm{mmHg}$ ) without antiglaucoma medication (average decrease $27.7 \pm 6.5 \mathrm{mmHg}$; range $16-41 \mathrm{mmHg}$ ), which was significant $(P<0.00001)$ compared with baseline. After an average follow-up period of $36.6 \pm 1.0$ months (range 35-38 months), the mean IOP stabilized at $17.4 \pm 2.2 \mathrm{mmHg}$ (range 12-21 mmHg). The nasal angle recess did not close again in any one of the patients during the follow-up period. The average significant $(P<0.00001)$ decrease in corneal endothelial cell density in the treated eyes was $260 \pm 183 \mathrm{cells} / \mathrm{mm}^{2}$ (range 191-328 cells $/ \mathrm{mm}^{2}$ ).

Conclusions: Anterior chamber paracentesis and GSL lowers IOP in advanced CACG, though it may lead to mild corneal endothelial cell loss.

Keywords: angle-closure glaucoma, surgery, intraocular pressure, treatment

\section{Introduction}

Glaucoma, the second leading cause of blindness worldwide, affects about 66.8 million people and causes bilateral blindness in 6.7 million individuals. ${ }^{1}$ In Asia, primary angleclosure glaucoma is the major form of the disease. Chronic angle-closure glaucoma (CACG) is highly prevalent in China, India, and other South-East Asian countries, in which about $40 \%$ of the world's population lives. ${ }^{2-15}$

Peripheral anterior synechiae (PAS) around the circumference of the anterior chamber (AC) angle is thought to cause CACG, which blocks aqueous humor outflow in the trabecular meshwork-Schlemm's canal pathway and the uveoscleral pathway. ${ }^{16-23}$ As PAS formation is a quiet and gradual process, intraocular pressure (IOP) in the affected eye may increase slowly. As a result, most patients with CACG are asymptomatic and tolerate the elevated IOP well until a very late stage of the disease. When patients notice 
any problem, they may already have severe visual disability, accounting for the high rate of blindness. ${ }^{6,9,18}$

Compared with primary open-angle glaucoma, the etiology of CACG seems simpler and more like an anatomic disorder. Adhesion between the peripheral iris and AC angle wall mechanically blocks the aqueous humor outflow from entering the trabecular meshwork and/or ciliary band, leading to IOP elevation and glaucomatous neuropathy. 6,8,9,11,14,16,18,19,21-24 Etiologic treatment, one of the most fundamental and important principles in medicine, has not been applied fully in the treatment of CACG, due to technical difficulties. Although it is widely accepted that PAS formation causes CACG, PAS dissection and reopening of the closed angle have not been emphasized as essential in the management of CACG. The treatment protocols for CACG are similar to those of primary open-angle glaucoma, which has far more complicated etiology and pathogenesis, except for peripheral laser iridotomy (PI) to relieve pupillary block and/or laser peripheral iridoplasty (LPI) to widen the AC angle before medication or surgery. $6,7,9,11,13,17,18,22,24,25$ Antiglaucoma medication is prescribed routinely after laser therapy if IOP exceeds $21 \mathrm{mmHg}$ or glaucomatous neuropathy or visual defect are confirmed. For advanced cases, filtration surgery, mostly trabeculectomy, is performed, because medication alone cannot decrease the elevated IOP to the target level.

PAS dissection has not been highlighted in the treatment of CACG, because of a few factors. First, PAS develops in the circumference of the $\mathrm{AC}$ angle and is difficult to access during surgery. In clinical practice, visualization of the AC angle can be achieved only with a goniolens, which covers the cornea and prevents intraocular procedures. To dissect PAS in the AC angle through the cornea, a modified goniolens is required, which aids the visualization of the angle but interferes with the manipulation of the dissection. This barrier might prevent surgeons from considering dissection of PAS as a treatment for CACG. Second, it is unclear whether the synechiae between the iris root and angle wall leads to functional impairment of the trabecular meshwork. If this is the case, PAS dissection will not benefit aqueous humor outflow and will be meaningless as a management strategy. Finally, many currently available antiglaucoma medications, ie, carbonic anhydrase inhibitors, $\beta$-blockers, and prostaglandin analogs, are effective in patients with $\mathrm{CACG},{ }^{24-29}$ and that success has dampened the enthusiasm to develop more rational treatments for CACG.

The goal of the current study was to evaluate the IOPlowering effect of PAS dissection on CACG with a novel slit-lamp procedure that treated $180^{\circ}$ of PAS.

\section{Materials and methods Patients}

CACG was defined as glaucomatous optic neuropathy with a compatible visual field defect or visual disability and at least $180^{\circ}$ of synechial angle closure on dynamic gonioscopy. CACG is a bilateral ocular disorder, although the morbidity and manifestations are usually asymmetric. It is common in clinical practice for a patient with CACG to have one severely damaged eye, referred to as the glaucomatous eye in the current study, while the fellow eye is mildly or not functionally affected at the initial diagnosis, referred to as the fellow eye in the current study.

The inclusion criteria included a best-corrected visual acuity (BCVA) of less than 20/200 in the glaucomatous eye. The exclusion criteria included a history of ocular trauma, inflammation, intraocular surgery, laser peripheral iridoplasty, an episode of acute angle-closure glaucoma, a ciliary cyst or tumor identified by ultrasound biomicroscopy, and age below 18 years or above 80 years.

\section{Surgical intervention}

In the current study, we introduced an innovative slit-lamp procedure, AC paracentesis and goniosynechialysis (GSL), as a surgical intervention. It is derived and developed from paracentesis, which is an established technique for lowering elevated IOP in acute angle-closure glaucoma, a similar ocular disorder secondary to a narrow or closed angle.

Before the procedure, the patients underwent detailed ophthalmologic examinations that included measurement of the VA, BCVA, biomicroscopy, and IOP; specular microscopy, fundus examination; gonioscopy; ultrasound biomicroscopy; and Humphrey visual field analysis if applicable. The width of the AC angle recess was graded in all four quadrants using Shaffer's classification system with dynamic gonioscopy.

In AC paracentesis and GSL, the needle was inserted into the $\mathrm{AC}$ and advanced to the opposite periphery over the iris plane. To avoid interpersonal surgical skill bias, the same doctor (GQ) performed AC paracentesis-guided GSL. To prepare the eyes for surgery, two drops of tobramycin $0.3 \%$ and two drops of sterile pilocarpine $2 \%$ were instilled in the surgical eye twice at an interval of 5 minutes. Three drops of sterile Alcaine (proparacaine, Alcon, Fort Worth, TX) was applied as topical anesthesia. The patient sat in front of the slit lamp with the beam focused on the nasal AC and iris. A 26-gauge needle with a syringe was inserted into the AC through the peripheral cornea of the inferotemporal quadrant. The needle tip was advanced to the inferior $\mathrm{AC}$ angle over 
the iris plane. To dissect the PAS, the needle tip was placed on the iris root with the bevel at the front side, which was visualized through the slip-lamp biomicroscope (Figure 1A). The surgeon then pushed the iris root backward to drag it down from the angle wall and trabecular meshwork surface (Figure 1B). The procedure began inferiorly at the 6 o'clock position and moved clockwise to the 9 o'clock position nasally. To separate the superonasal quadrant, the needle was withdrawn and reinserted into the $\mathrm{AC}$ at the peripheral cornea of the superotemporal quadrant. PAS dissection in the superonasal quadrant started at the 9 o'clock position and moved clockwise to the 12 o'clock position superiorly. Care must be taken to avoid touching the crystalline lens when pushing the nasal iris root near the 9 o'clock position. Four to five pushes were done in each quadrant to dissect the PAS. At the completion of the procedure, tobramycin and dexamethasone ointment (Tobradex, Alcon) were instilled into the conjunctival sac before the eye was patched. Postoperative care included instillation of tobramycin and dexamethasone eye drops four times daily from postoperative days 1 to 7 .

The patients were examined on postoperative days 1 and 7, 1 month, and every 6 months. PI was performed in both the glaucomatous eye and the fellow eye to relieve pupillary block at the second follow-up visit 1 week after GSL. Antiglaucoma medication was prescribed if the postoperative IOP exceeded $21 \mathrm{mmHg}$.
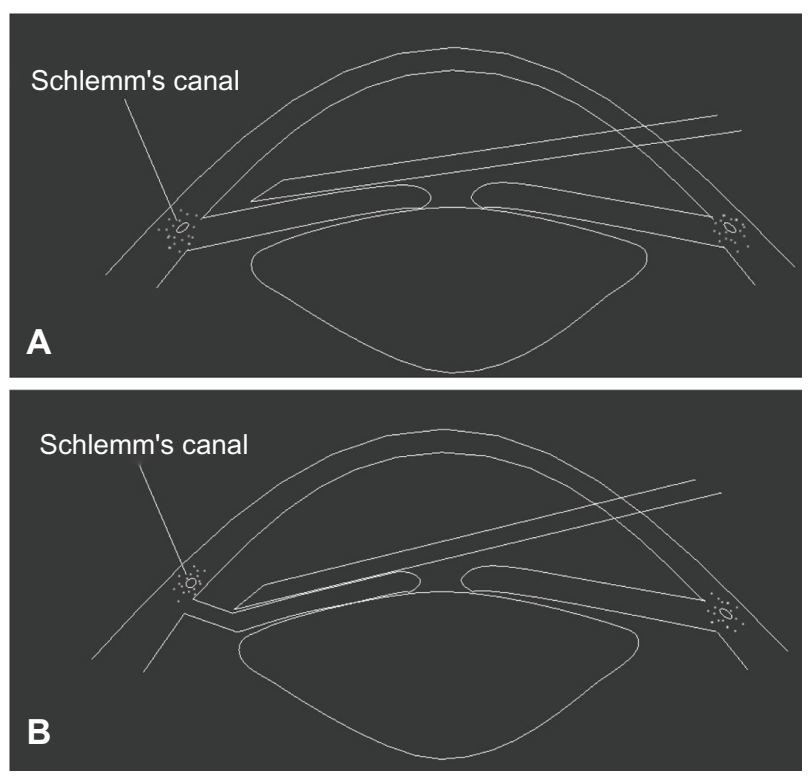

Figure I (A) A 26-gauge needle is inserted into the anterior chamber angle in front of the iris before peripheral anterior synechiae dissection. (B) The surgeon pushed the iris root backward to drag it down from the angle wall and trabecular meshwork surface.

Note: The trabecular meshwork is exposed after the dissection of the peripheral anterior synechiae.

\section{Primary outcomes}

The follow-up examinations included measurement of VA, BCVA, and IOP; biomicroscopy; gonioscopy at each visit; and measurement of the corneal endothelial cell count at the last visit. Postoperative IOP, corneal endothelial cell density, and angle status on gonioscopy were the primary outcome measurements.

\section{Statistical analysis}

The means of postoperative IOP 1 week later and corneal endothelial cell density counted at the last follow-up visit were compared with baseline with a paired sample $t$-test. The significance level was set at 5\%. All statistical analyses were carried out using Statistical Package for the Social Sciences version 12.0 (SPSS, Chicago, IL).

\section{Clinical trial registration}

Clinical trial registration of this study was done on the Chinese Clinical Trial Registry website (http://www.chictr.org), where the registration information is publicly available.

\section{Statement of ethics}

This study was conducted in Beijing Tongren Eye Center, Beijing Tongren Hospital, Capital Medical University, Beijing, China, after the institutional review board and ethics committee approved the study. All patients provided informed consent after detailed information about the procedure was provided.

\section{Results}

Thirty patients (18 men, 12 women) were diagnosed with CACG and enrolled from November 2007 to February 2008 at the Beijing Tongren Eye Center. Each patient had a glaucomatous eye that was severely affected and a fellow eye that was mildly affected or functionally normal. The average patient age was $53.1 \pm 12.6$ years (range $28-74$ years). At the initial diagnosis, the average IOP values of the glaucomatous eyes and the fellow eyes were $47.1 \pm 6.7 \mathrm{mmHg}$ (range 39-61 $\mathrm{mmHg}$ ) and $19.3 \pm 4.8 \mathrm{mmHg}$ (range 11-29 $\mathrm{mmHg}$ ), respectively. After limited GSL, the IOP of the glaucomatous eyes decreased dramatically to $24.5 \pm 5.0 \mathrm{mmHg}$ (range $17-38 \mathrm{mmHg}$ ) on postoperative day 1 and $19.3 \pm 2.8 \mathrm{mmHg}$ (range 14-26 mmHg) 1 week later. The average decrease in IOP of the glaucomatous eyes after treatment was $27.7 \pm 6.5 \mathrm{mmHg}$ (range 16-41 mmHg), which was significantly different compared with that at diagnosis ( $P=0.000$, paired samples $t$-test). At the last follow-up visit (average 36.6 \pm 1.0 months; range 35-38 months), the mean 
IOP of the glaucomatous eyes was $17.4 \pm 2.2 \mathrm{mmHg}$ (range $12-21 \mathrm{mmHg}$ ). Nine patients were receiving pilocarpine as an adjunctive medication, because the follow-up IOP increased to $21 \mathrm{mmHg}$ or higher. Following the medical treatment, the IOP decreased into the teens. Daily pilocarpine eye drops were also prescribed for patient 7 , who had the highest IOP of $21 \mathrm{mmHg}$ at the last visit. She had had stable postoperative IOP below $21 \mathrm{mmHg}$, but IOP increased to $21 \mathrm{mmHg}$ only at the last visit.

Synechial angle closure of $360^{\circ}$ was seen in the AC angle of all eyes that underwent GSL in which the trabecular meshwork band was invisible on dynamic gonioscopy. After AC paracentesis and GSL were performed for $180^{\circ}$ nasally, the trabecular meshwork band in the nasal quadrants was exposed. Remnant pigment granules were commonly seen on Schwalbe's line and the anterior trabecular meshwork surface (Figure 2). No recurrence of PAS formation or angle closure was discerned in the nasal angle of the treated eyes during the study period.

No cataract formation or decrease in VA or BCVA was revealed in any one of the treated eyes posterior to limited GSL. The mean pretreatment corneal endothelial cell density of the glaucomatous eyes was $2318 \pm 509$ cells $/ \mathrm{mm}^{2}$ (range $1423-2980$ cells $/ \mathrm{mm}^{2}$ ), which decreased to $2060 \pm 468$ cells $/ \mathrm{mm}^{2}$ (range $1167-2865$ cell $/ \mathrm{mm}^{2}$ ) at follow-up 36.6 \pm 1.0 months (range 35-38 months) later. The average decrease in the corneal endothelial cell density was $260 \pm 183$ cells $/ \mathrm{mm}^{2}$ (range 16-754 cells $/ \mathrm{mm}^{2}$ ), which was statistically significant $(P=0.000, t=7.753$, paired samples $t$-test). The average pre-PI and post-PI corneal endothelial cell counts of the fellow eyes were $2513 \pm 251$ cells $/ \mathrm{mm}^{2}$ (range 1477-3012 cells $/ \mathrm{mm}^{2}$ ) and $2511 \pm 267$ cells $/ \mathrm{mm}^{2}$ (range 1478-3010 cells $/ \mathrm{mm}^{2}$ ), respectively, which did not differ significantly.

Intermittent ocular pain was the most common symptom in the glaucomatous eyes among the enrolled patients. Twenty-two of $30(73.3 \%)$ patients reported ocular pain before the procedure. The pain resolved in all cases during the postoperative follow-up period when the IOP decreased.

AC bleeding was a common intraoperative complication, occurring in $25(83.3 \%)$ of 30 patients. Because bleeding was usually minimal and easily stopped with application of pressure to the outer upper eyelid when the needle was withdrawn, the procedure was completed in all patients. The bleeding did not require special care or medication and was resolved within a few days. No other complications or safety problems developed intraoperatively or during the follow-up period.
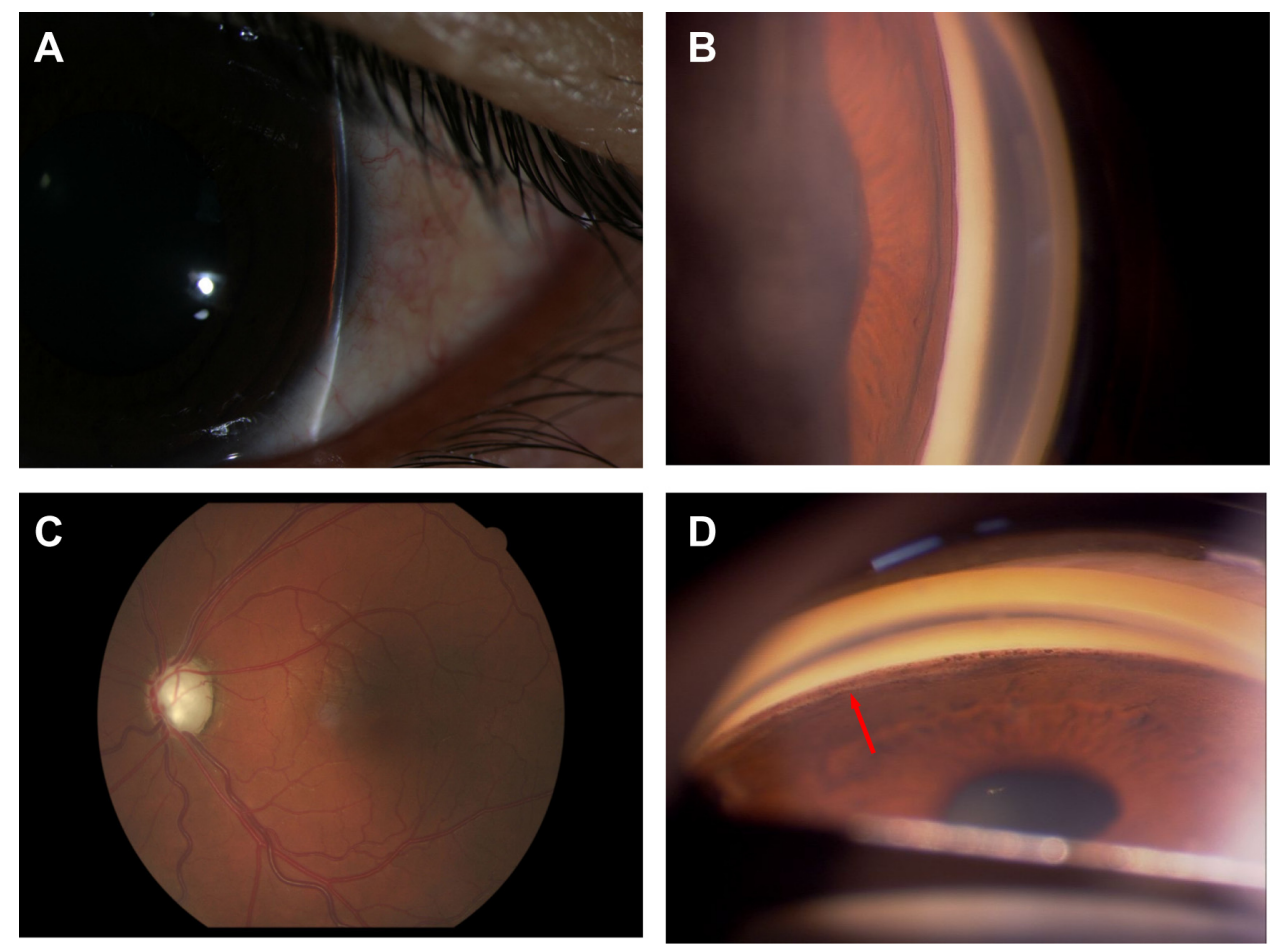

Figure 2 (A) Peripheral anterior chamber angle width assessment using the Von Herrick method on biomicroscopy in the left eye of patient 3. (B) Dynamic gonioscopy shows that the nasal angle is closed due to massive peripheral anterior synechiae. (C) The cup of the optic nerve head in the left eye is enlarged to the edge of the disc (vertical cup/disc ratio of I.0). (D) The trabecular meshwork is exposed after the procedure (arrow), with remnant pigment granules on the Schwalbe's line and trabecular meshwork. 


\section{Discussion}

We evaluated the IOP-lowering effect of PAS dissection and reopening of the closed angle on CACG through a new and uncomplicated procedure, paracentesis-guided limited GSL, performed with a slit lamp in 30 eyes of 30 patients with CACG. The current results were impressive, in that all treated eyes achieved a substantial average decrease in IOP of $27.7 \pm 6.5 \mathrm{mmHg}$ (range 16-41 $\mathrm{mmHg}$ ). No safety problems were observed with this procedure except for mild corneal endothelial cell loss.

PAS dissection and/or closed AC angle widening had been tried as a treatment for CACG but were usually conducted as adjunct procedures with other intraocular surgeries, mostly phacoemulsification. ${ }^{30-34}$ As a result, it was hard to evaluate the IOP-lowering efficacy of PAS dissection or angle-widening procedures on CACG. LPI is a simpler and more direct laser therapy intended to reopen the closed angle by dissecting the PAS through peripheral iris contraction. ${ }^{18,22,35-37}$ The laser burns in the peripheral iris result in iris contraction that pulls the iris posteriorly away from the trabecular meshwork and angle wall and opens the closed angle. As the iris tissue contraction is usually too weak to dissect established PAS, LPI has limited efficacy in patients with CACG. ${ }^{22,38}$

The use of AC paracentesis and GSL in the current study is a novel way to dissect PAS by separating the peripheral iris from the anterior $\mathrm{AC}$ angle wall by pushing the iris root back. It is superior to LPI laser burns in power and has showed excellent efficiency in dissecting PAS and reopening the angle recess, as shown by the gonioscopy results. Except for dissecting the PAS and reopening the closed angle, there were no other side effects of this slit-lamp procedure on the AC structures. Therefore, it was easier to evaluate the IOPlowering efficacy of GSL on CACG. Considering that the IOP decreased dramatically in all treated eyes and only the nasal $180^{\circ}$ of the angle was reopened, we concluded that PAS dissection effectively lowered IOP in patients with CACG, and that synechial angle closure in CACG does not necessarily result in functional impairment of the trabecular meshwork or the aqueous humor outflow pathway.

We tested our hypothesis in eyes with advanced CACG, because, if GSL works in patients with end-stage CACG, it is likely that it will be effective in mild cases, because PAS is usually wider and more established in severe cases. Conversely, if PAS dissection lowers the IOP in patients with early-stage CACG, that does not necessarily mean that the procedure will be effective in cases with advanced or end-stage CACG. The latter would have had more serious trabecular meshwork damage and functional impairment if PAS formation does lead to pathological changes and functional disability to aqueous humor outflow pathway.

In the current study, no serious intraoperative or postoperative complications developed except for minimal bleeding in the $\mathrm{AC}$, which did not prevent completion of the surgery and resolved spontaneously during the first postoperative days. Although it turned out to be an effective and successful method to dissect PAS in patients with CACG, and no serious complications like endophthalmitis and cataract formation were seen in the operated eyes, we still want to warn that $\mathrm{AC}$ paracentesis-guided GSL is a potentially risky intraocular procedure. The inherent risks for slit-lamp procedures, such as it being difficult to maintain a sterile field, difficult to control the patient's head position, and difficult to adjust focus during the performance, may impact on the procedure's generalization. In this study, we used it just as a resolution for PAS dissection. And it is not one of our purposes to recommend or generalize this slit-lamp procedure for other surgeons.

To the best of our knowledge, AC paracentesis-guided GSL has never been reported in the literature as a treatment for CACG. There is no standard method of performing this novel technique. We developed this procedure from AC paracentesis and mastered the technique after performing it numerous times in CACG patients. It is not problematic to perform it from the temporal side of the cornea and separate the nasal angle. However, separating the temporal angle from the nasal cornea is much more difficult because the patient's nose may interfere with the surgical manipulations during the procedure. Considering the difficulties of the procedure and uncertainties of the results, we dissected the PAS only in the nasal $180^{\circ}$ of the angle, which was unlikely to cause safety issues if the treatment did not reduce the IOP in the affected eyes.

A limitation of the current study was that it was a noncomparative pilot study. The long-term outcome remains unclear, as ten patients were prescribed adjunctive pilocarpine eye drops due to an IOP increase after 3 years of follow-up. Further research, especially a randomized, multicenter, prospective, controlled study, is needed before this new slit-lamp procedure can be accepted as a treatment for CACG.

\section{Conclusion}

In summary, based on the current study, we concluded tentatively that GSL through PAS dissection effectively lowers IOP in patients with CACG, though it may cause mild corneal endothelial cell loss. 


\section{Acknowledgments}

Meeting presentation: oral presentation at the World Ophthalmology Congress in Abu Dhabi, UAE, February 20, 2012. This work was supported by the Beijing Municipal Health Bureau High-level Medical Professionals Promotion Project (2011-3-044), Ministry of Science and Technology of China grant (2010IM030800), and National Nature Science Foundation of China grants $(30921064,90820307)$.

\section{Disclosure}

The authors declare no conflict of interest.

\section{References}

1. Henson DB, Thampy R. Preventing blindness from glaucoma. $B M J$. 2005;331(7509):120-121.

2. Wang YX, Xu L, Yang H, Jonas JB. Prevalence of glaucoma in North China: the Beijing Eye Study. Am J Ophthalmol. 2010;150(6): 917-924.

3. Song W, Sun X, Shao Z, et al. Prevalence and causes of visual impairment in a rural North-east China adult population: a population-based survey in Bin County, Harbin. Acta Ophthalmol. 2011;88(6):669-674.

4. Huang S, Zheng Y, Foster PJ, Huang W, He M. Prevalence and causes of visual impairment in Chinese adults in urban southern China. Arch Ophthalmol. 2009;127(10):1362-1367.

5. Li Z, Cui H, Liu P, Zhang L, Yang H, Zhang L. Prevalence and causes of blindness and visual impairment among the elderly in rural southern Harbin, China. Ophthalmic Epidemiol. 2008;15(5):334-338.

6. He M, Foster PJ, Johnson GJ, Khaw PT. Angle-closure glaucoma in East Asian and European people. Different diseases? Eye (Lond). 2006; 20(1):3-12.

7. Sihota R, Agarwal HC. Profile of the subtypes of angle closure glaucoma in a tertiary hospital in north India. Indian J Ophthalmol. 1998;46(1):25-29.

8. Sood D, Sood NN. Angle closure and India. Indian J Ophthalmol. 2006;54(3):147-148.

9. Chew PT, Aung T. Primary angle-closure glaucoma in Asia. J Glaucoma. 2001;10(5 Suppl 1):S7-S8.

10. Foster PJ, Oen FT, Machin D, et al. The prevalence of glaucoma in Chinese residents of Singapore: a cross-sectional population survey of the Tanjong Pagar district. Arch Ophthalmol. 2000;118(8):1105-1111.

11. Kumar RS, Baskaran M, Chew PT, et al. Prevalence of plateau iris in primary angle closure suspects an ultrasound biomicroscopy study. Ophthalmology. 2008;115(3):430-434.

12. Kumar RS, Tantisevi V, Wong MH, et al. Plateau iris in Asian subjects with primary angle closure glaucoma. Arch Ophthalmol. 2009;127(10):1269-1272.

13. See JL, Chew PT. Glaucoma in Singapore. J Glaucoma. 2004;13(5): 417-420.

14. Shen SY, Wong TY, Foster PJ, et al. The prevalence and types of glaucoma in malay people: the Singapore Malay eye study. Invest Ophthalmol Vis Sci. 2008;49(9):3846-3851.

15. Sim DH, Goh LG, Ho T. Glaucoma pattern amongst the elderly Chinese in Singapore. Ann Acad Med Singapore. 1998;27(6):819-823.

16. Nongpiur ME, Ku JY, Aung T. Angle closure glaucoma: a mechanistic review. Curr Opin Ophthalmol. 2011;22(2):96-101.

17. Nazm N, Gandhi M, Dubey S, Pegu J. Angle closure glaucoma. Ophthalmology. 2009;116(12):2478; author reply 2478-2479.

18. Amerasinghe N, Aung T. Angle-closure: risk factors, diagnosis and treatment. Prog Brain Res. 2008;173:31-45.

19. He M, Lu Y, Liu X, Ye T, Foster PJ. Histologic changes of the iris in the development of angle closure in Chinese eyes. J Glaucoma. 2008;17(5):386-392.
20. Friedman DS, Vedula SS. Lens extraction for chronic angle-closure glaucoma. Cochrane Database Syst Rev. 2006;3:CD005555.

21. Salmon JF, Sharma T. Chronic angle-closure glaucoma. Ophthalmology. 2005;112(10): 1844

22. Rosman M, Aung T, Ang LP, Chew PT, Liebmann JM, Ritch R. Chronic angle-closure with glaucomatous damage: long-term clinical course in a North American population and comparison with an Asian population. Ophthalmology. 2002;109(12):2227-2231.

23. Aung T, Lim MC, Chan YH, Rojanapongpun P, Chew PT. Conuration of the drainage angle, intraocular pressure, and optic disc cupping in subjects with chronic angle-closure glaucoma. Ophthalmology. 2005;112(1):28-32.

24. Aung T, Chan YH, Chew PT. Degree of angle closure and the intraocular pressure-lowering effect of latanoprost in subjects with chronic angleclosure glaucoma. Ophthalmology. 2005;112(2):267-271.

25. Sihota R, Saxena R, Agarwal HC, Gulati V. Crossover comparison of timolol and latanoprost in chronic primary angle-closure glaucoma. Arch Ophthalmol. 2004;122(2):185-189.

26. Cheng JW, Cai JP, Li Y, Wei RL. A meta-analysis of topical prostaglandin analogs in the treatment of chronic angle-closure glaucoma. J Glaucoma. 2009;18(9):652-657.

27. How AC, Kumar RS, Chen YM, et al. A randomised crossover study comparing bimatoprost and latanoprost in subjects with primary angle closure glaucoma. Br J Ophthalmol. 2009;93(6):782-786.

28. Chen MJ, Chen YC, Chou CK, Hsu WM. Comparison of the effects of latanoprost and travoprost on intraocular pressure in chronic angle-closure glaucoma. J Ocul Pharmacol Ther. 2006;22(6): 449-454.

29. Sakai H, Shinjyo S, Nakamura Y, Nakamura Y, Ishikawa S, Sawaguchi S. Comparison of latanoprost monotherapy and combined therapy of $0.5 \%$ timolol and $1 \%$ dorzolamide in chronic primary angle-closure glaucoma (CACG) in Japanese patients. J Ocul Pharmacol Ther. 2005;21(6):483-489.

30. Lai JS, Tham CC, Chua JK, Lam DS. Efficacy and safety of inferior 180 degrees goniosynechialysis followed by diode laser peripheral iridoplasty in the treatment of chronic angle-closure glaucoma. J Glaucoma. 2000;9(5):388-391.

31. Lai JS, Tham CC, Lam DS. The efficacy and safety of combined phacoemulsification, intraocular lens implantation, and limited goniosynechialysis, followed by diode laser peripheral iridoplasty, in the treatment of cataract and chronic angle-closure glaucoma. J Glaucoma. 2001;10(4):309-315.

32. Kiuchi Y, Tsujino C, Nakamura T, Otori Y, Mochizuki H. Phacoemulsification and trabeculotomy combined with goniosynechialysis for uncontrollable chronic angle-closure glaucoma. Ophthalmic Surg Lasers Imaging. 2011;41(3):348-354.

33. Papamatheakis DG, Devaux A, Cordahi G, Harasymowycz PJ. Chronic angle-closure glaucoma secondary to a suprachoroidal effusion induced by central retinal vein occlusion. Ophthalmic Surg Lasers Imaging. 2007;38(3):248-249.

34. Teekhasaenee C, Ritch R. Combined phacoemulsification and goniosynechialysis for uncontrolled chronic angle-closure glaucoma after acute angle-closure glaucoma. Ophthalmology. 1999;106(4):669-674; discussion 674-675.

35. Sawada A, Aoyama A, Yamamoto T, Takatsuka N. Long-term therapeutic outcome of acute primary angle closure in Japanese. Jpn J Ophthalmol. 2007;51(5):353-359.

36. Lam DS, Tham CC, Lai JS, Leung DY. Current approaches to the management of acute primary angle closure. Curr Opin Ophthalmol. 2007;18(2):146-151.

37. Lai JS, Tham CC, Chua JK, Poon AS, Lam DS. Laser peripheral iridoplasty as initial treatment of acute attack of primary angleclosure: a long-term follow-up study. J Glancoma. 2002;11(6): 484-487.

38. Agarwal HC, Kumar R, Kalra VK, Sood NN. Argon laser iridoplasty: a primary mode of therapy in primary angle closure glaucoma. Indian J Ophthalmol. 1991;39(3):87-90. 
Clinical Ophthalmology

\section{Publish your work in this journal}

Clinical Ophthalmology is an international, peer-reviewed journal covering all subspecialties within ophthalmology. Key topics include: Optometry; Visual science; Pharmacology and drug therapy in eye diseases; Basic Sciences; Primary and Secondary eye care; Patient Safety and Quality of Care Improvements. This journal is indexed on

PubMed Central and CAS, and is the official journal of The Society of Clinical Ophthalmology (SCO). The manuscript management system is completely online and includes a very quick and fair peer-review system, which is all easy to use. Visit http://www.dovepress.com/ testimonials.php to read real quotes from published authors. 